\title{
ON THE MOTION OF A MAGNETICALLY CONFINED PLASMA CYLINDER*
}

\author{
BY \\ CHONG-WEI CHU** \\ University of Minnesota, Minneapolis, Minnesota
}

1. Introduction. Owing to the recent interest in controlled nuclear fusion, the stability of a linear pinch, a magnetically confined plasma cylinder, has been a subject of rather extensive studies [1], [2], [3]. Theoretically it was found possible to find a stabilized linear pinch under some simplifying assumptions. Practically, however, complete stabilization of a pinch has not yet been achieved.

In a stability analysis one usually considers small perturbations from the equilibrium configuration. Since a pinch device can hardly produce a regular-shaped pinch, the perturbation is certainly not always small. It is, therefore, advisable to investigate the motion of a pinch due to finite disturbances.

The aim of this paper is two-fold. First, it investigates the stability of a linear pinch under finite axisymmetric disturbances. Secondly, it studies the motion of a magnetically confined plasma cylinder, a problem of considerable mathematical interest.

The magnetohydrodynamic approach to plasma problems will be adopted, and the plasma is assumed to be inviscid, non-heat-conducting, and to have infinite electrical conductivity. A one-dimensional model of a linear pinch will be proposed with a derivation of its governing partial differential equations, which turn out to be of mixed type. The conditions leading to trivial solutions and simple exact solutions of the differential equations are then studied. There follows a treatment of the nonlinear wave motion in the hyperbolic region. For the elliptic region the maximum principle is applied to give some conditions for instability. Finally the hodograph transformation is presented, and a method of finding infinitely many particular solutions of the hodograph equation is given.

2. One-dimensional model and differential equations. The one-dimensional model we shall study is an infinite cylindrical plasma confined by an azimuthal magnetic field; the cylindrical plasma has an internal magnetic field which is initially uniform and axial. We will consider finite axisymmetric disturbances with small area gradient in the axial direction; so the internal field will remain essentially axial and approximately uniform across the plasma cylinder, for magnetic lines are material lines in a perfectly conducting fluid flow [4]. Since the magnetic flux through any moving material surface in a perfectly conducting medium remains constant [4], the internal magnetic field $B_{i}$ is inversely proportional to the cross-sectional area $a$;

$$
B_{i}=Q_{0} B_{0} / Q,
$$

where the subscript 0 indicates initial quantities. The confining magnetic field $B_{c}$ which is generated by a constant axial surface current, is purely azimuthal. By Ampère's law it is inversely proportional to the radial distance $r$ :

*Received May 29, 1963; revised manuscript received Aug. 9, 1963. This paper is part of a Ph.D. thesis submitted to the faculty of the graduate school of the University of Minnesota (1963). This work was supported by the U. S. Air Force under contract monitored by the Aeronautical Research Laboratories, Wright Air Development Division.

**Now with Northrop Norair, Hawthorne, Cal. 


$$
B_{c}=c / r=C_{0} R_{0} / r,
$$

where $c$ is a constant; $R_{0}$ is the initial equilibrium radius, and $C_{0}$ is the initial confining magnetic field on the surface. Across the interface between the plasma and the confining magnetic field, the sum of the plasma pressure $p$ and the magnetic pressure $\mu_{0}^{-1} B^{2} / 2$ is continuous [4], where the rational MKS system of units is adopted and $\mu_{0}$ is the permeability of the vacuum. Applying this boundary condition, and setting $A=Q / Q_{0}$ and $\eta=\left(B_{0} / C_{0}\right)^{2}$, we obtain

$$
p=\frac{1}{2} \mu_{0}^{-1} A^{-2}\left(C_{0}^{2} A-B_{0}^{2}\right)=p_{0}(1-\eta)^{-1} A^{-2}(A-\eta) .
$$

Note that $1>\eta$ and $A>\eta$ since $p>0$.

With all dissipations neglected, the isentropic relation holds; hence

$$
\rho=\rho_{0}\left(p / p_{0}\right)^{1 / \gamma},
$$

where $\rho$ is the density of the plasma and $\gamma$ is the isentropic component. It is assumed that at each cross-section representative physical quantities may be assigned through some process of averaging so that the flow may be considered one-dimensional. In this case the equation of continuity, taking account of the time dependence of the cross-sectional area, is [5]

$$
(\rho A)_{t}+(\rho u A)_{x}=0,
$$

where $u$ is the flow velocity. Moreover, under the assumption that all disturbances considered have small area gradients in the axial direction, the axial Lorentz force is negligibly small and the momentum equation is simply [5]

$$
\rho u_{t}+\rho u u_{z}=-p_{x} \text {. }
$$

Eliminating $p$ and $\rho$ from (1), (2), (3), and (4), we finally obtain

$$
\begin{aligned}
& u_{t}+u u_{x}+f(A) A_{x}=0, \\
& A_{t}+u A_{x}+g(A) u_{x}=0,
\end{aligned}
$$

where

$$
\begin{gathered}
f(A)=p_{0} \rho_{0}^{-1}(1-\eta)^{(1-\gamma) / \gamma}(A-\eta)^{-1 / \gamma}(2 \eta-A) A^{(2-3 \gamma) / \gamma}, \\
g(A)=\gamma A(A-\eta)[(\gamma-1) A+(2-\gamma) \eta]^{-1} \geqq 0 .
\end{gathered}
$$

Note that (5) and (6) are of mixed type: hyperbolic for $\eta<A<2 \eta$, but elliptic for $2 \eta<A$.

3. Simple cases. A few simple cases, where some of the derivatives vanish identically, will now be studied.

Theorem 3.1. Let $u=u(x, t)$ and $A=A(x, t)$ be a solution of (5) and (6) in a region $R$. Suppose any pair of the partial derivatives $u_{t}, u_{x}, A_{t}$, and $A_{x}$ vanishes identically over $R$. Then $u \equiv$ constant and $A \equiv$ constant in $R$.

Proof. The proof is trivial except for the case $A_{t}=0$ and $u_{t}=0$. Now setting $A_{t}=0$ and $u_{t}=0$ in (5) and (6) gives

$$
\begin{aligned}
& u u_{x}+f(A) A_{x}=0 \\
& u A_{x}+g(A) u_{x}=0 .
\end{aligned}
$$

If there is a non-trivial solution, it must be true that $u^{2}-f(A) g(A)=0$. Differentiating 
this equation and comparing with (5a), we obtain

$$
\left(2+g^{\prime}\right) f=-f^{\prime} g,
$$

which contradicts (7). Hence only trivial solutions exist.

Theorem 3.2. A necessary and sufficient condition that (5) and (6) have only trivial solutions $u=$ const and $A=$ const is $u_{x} \equiv 0$.

Proof. The proof of necessity is obvious. To prove sufficiency, we set $u_{x}=0$ in (5) and (6) to obtain

$$
\begin{gathered}
\frac{d u}{d t}+f(A) A_{x}=0, \\
A_{\iota}+u A_{x}=0 .
\end{gathered}
$$

Elimination of $A_{x}$ from (5b) and (6b) yields $f(A) A_{t}-u d u / d t=0$ which, after integration with respect to $t$, gives

$$
\int f(A) d A-\frac{1}{2} u^{2}=C(x) .
$$

Differentiating the above equation with respect to $x$, we find $f(A) A_{x}=C^{\prime}(x)$, which implies

$$
\frac{d u}{d t}=-f(A) A_{x}=\text { const }=C_{1}
$$

on account of $(5 \mathrm{~b})$. We assert that $C_{1}=0$, and hence only trivial solutions exist. Indeed if $C_{1} \neq 0$, then $A=2 \eta$ is attained at some $x$, say $x=x^{\prime}$, since $A_{x}$ can not change sign except at $A=2 \eta$. Noting the sigh change of $f(A)$ at $x=x^{\prime}$, we see that

$$
\begin{array}{lll}
\lim _{x \rightarrow x^{\prime}}\left[f(A) A_{x}\right]>0 & \text { which implies } C_{1}<0, \text { but } \\
\lim _{x \rightarrow x^{\prime}}\left[f(A) A_{x}\right]<0 & \text { which implies } C_{1}>0 .
\end{array}
$$

The assumption $C_{1}=0$ leads to a contradiction; hence $C_{1}=0$.

Theorem 3.3. A plasma cylinder whose motion is governed by (5) and (6) can not maintain its cylindrical shape (i.e., $A_{x} \not \equiv 0$ ) under a finite disturbance.

Proof. If $A_{x} \equiv 0$ under a finite disturbance, (5) and (6) reduce to

$$
\begin{gathered}
u_{t}+u u_{x}=0, \\
\frac{d A}{d t}+g(A) u_{x}=0 .
\end{gathered}
$$

Eliminating $u_{x}$, integrating with respect to $t$, and differentiating the resulting equation with respect to $x$, we obtain

$$
u_{x}=C_{1}(x) u .
$$

Substituting (8) in (5c) and integrating, we find

$$
u(x, t)=\left[C_{2}(x)+C_{1}(x) t\right]^{-1}=\left[C_{1}(x)\left(t+t_{0}\right)\right]^{-1},
$$

where $t_{0}$ is a constant independent of $x$ since on substituting (8a) into (8) the resulting $u_{x}$ must be independent of $x$ on account of (6c). For (8) and (8a) to be compatible, we must have $C_{1}^{\prime}(x)+C_{1}^{2}=0$, or $C_{1}(x)=\left(x+x_{0}\right)^{-1}$, where $x_{0}$ is a constant. We thus obtain the relation

$$
u(x, t)=\left(x+x_{0}\right)\left(t+t_{0}\right)^{-1},
$$


which leads to a contradiction since $u(x, t)$ is not finite $\lim _{|x| \rightarrow \infty} u(x, t) \rightarrow \infty$. The theorem is proved.

Note that (8b) is the simplest exact solution of (5) and (6) besides trivial solutions. To find $A$, we substitute (8b) into (6c) and integrate to obtain $\int[g(A)]^{-1} d A=-\ln \left(t+t_{0}\right)$, which integrates to

$$
A^{2-\gamma}(1-\eta)(A-\eta)^{-1}=\left[1+\left(t / t_{0}\right)\right]^{\gamma} .
$$

4. Wave motion in the hyperbolic region. In the hyperbolic region $\eta<A<2 \eta$ wave propagation is possible, for small disturbances may propagate along characteristics. Applying the theory of characteristics [6], we find the physical characteristics

$$
\begin{aligned}
& C_{+}:\left(\frac{d x}{d t}\right)_{+}=u+a(A), \\
& C_{-}:\left(\frac{d x}{d t}\right)_{-}=u-a(A),
\end{aligned}
$$

where

$$
\begin{aligned}
a(A)=[f(A) g(A)]^{1 / 2} & \\
& =\left(\frac{\gamma p_{0}}{\rho_{0}}\right)^{1 / 2} A^{(1-\gamma) / \gamma}\left(\frac{A-\eta}{1-\eta}\right)^{(\gamma-1) / 2 \gamma}\left[\frac{2 \eta-A}{(\gamma-1) A+(2-\gamma) \eta}\right]^{1 / 2}
\end{aligned}
$$

is the speed of propagation of disturbances relative to the medium. In addition, the hodograph characteristics are

$$
\begin{aligned}
& \Gamma_{+}: u+l(A)=2 r, \\
& \Gamma_{-}: u-l(A)=-2 s,
\end{aligned}
$$

where $r$ and $s$ are Riemann variables and

$$
l(A)=\int[f(A) / g(A)]^{1 / 2} d A .
$$

Definition. A simple wave is a flow whose hodograph image is a single $\Gamma$ characteristic [6].

It can be shown that a forward-facing (or backward-facing) simple wave lies on a single $\Gamma_{-}\left(\right.$or $\Gamma_{+}$) characteristic and has straight $C_{+}$(or $C_{-}$) characteristics [6].

The following theorem shows the effect of the ratio of the internal magnetic field to the confining magnetic field on the disturbance propagation speed.

Theorem 4.1. Let a be the speed of propagation defined by $(10 a)$. Then there exists a certain $A=A^{\prime}(\gamma, \eta)$ in the hyperbolic region $\eta<A<2 \eta$ such that

$$
\begin{array}{rcc}
a_{\eta}<0 & \text { for } & \eta<A<A^{\prime}, \\
=0 & & A=A^{\prime}, \\
>0 & A^{\prime}<A<2 \eta .
\end{array}
$$

Moreover, $A^{\prime}$ is the root of

$$
(\gamma-1)(A-1)(2 \eta-A)[(\gamma-1) A+(2-\gamma) \eta]+\gamma^{2} A(1-\eta)(A-\eta)=0
$$

in the interval $(\eta, 1)$. 
Proof. Differentiating (10a) with respect to $\eta$ gives

$$
a_{\eta}=\frac{a}{2 \gamma}\left\{\frac{(\gamma-1)(A-1)}{(1-\eta)(A-\eta)}+\frac{\gamma^{2} A}{(2 \eta-A)[(\gamma-1) A+(2-\gamma) \eta]}\right\} \text {. }
$$

Clearly, $a_{\eta}=0$ when

$N(A)=(\gamma-1)(A-1)(2 \eta-A)[(\gamma-1) A+(2-\gamma) \eta]+\gamma^{2} A(1-\eta)(A-\eta)=0$, which has one and only one root in $(\eta, 2 \eta)$ since $N(-\infty)>0, N(\eta)<0, N(1)>0$, $N(2 \eta)>0$ and $N(\infty)<0$. Let this root be $A=A^{\prime}$; we find that $a_{\eta}<0$ for $A<A^{\prime}$ but $a_{\eta}>0$ for $A>A^{\prime}$, and $A^{\prime}$ actually lies in the interval $(\eta, 1)$. Hence the theorem is proved.

An immediate consequence of Theorem 4.1 is:

Corollary. $a_{\eta}>0$ when $1 \leqq A<2 \eta$.

This means that the propagating speed of a disturbance with area (or hence pressure as seen from Eq. 1) greater than the initial equilibrium area (or pressure) increases with the initial ratio of the internal magnetic field to the confining magnetic field on the plasma surface.

One of the most conspicuous phenomena in nonlinear wave motion is the change of wave form embodied in the following theorems.

Theorem 4.2. There exists a critical $A=A_{c}(\gamma, \eta)$ in the hyperbolic region $\eta<A<2 \eta$ such that

$$
\begin{array}{rlc}
\left(\frac{d V}{d u}\right)_{-}>0 & \text { for } & \eta<A<A_{c}, \\
& =0 & A=A_{c}, \\
& <0 & A_{c}<A<2 \eta,
\end{array}
$$

where $(V)_{-}=u \pm a$ is the simple wave velocity.

Proof. Consider a forward-facing simple wave in which $u-l(A)=$ const. Then the simple wave velocity $(V)_{+}$is given by

$$
(V)_{+}=\left(\frac{d x}{d t}\right)_{+}=u+a(A)=a(A)+l(A)+\text { const. }
$$

Differentiating the above and using (10a), (11a) and (7), we obtain

$$
\left(\frac{d V}{d A}\right)_{+}=\frac{a(A)}{2 \gamma A}\left\{\frac{(\gamma-1) A+2 \eta}{A-\eta}-\frac{\gamma^{2} \eta A}{(2 \eta-A)[(\gamma-1) A+(2-\gamma) \eta]}\right\},
$$

which vanishes at least once for $\eta<A<2 \eta$ since

$$
\lim _{A \rightarrow \eta_{+}}\left(\frac{d V}{d A}\right)_{+} \rightarrow+\infty, \quad \lim _{A \rightarrow 2 \eta_{-}}\left(\frac{d V}{d A}\right)_{+} \rightarrow-\infty .
$$

Clearly, for $\eta<A<2 \eta$ the derivative $(d V / d A)_{+}$vanishes when

$$
N(A)=(2 \eta-A)[(\gamma-1) A+2 \eta)][(\gamma-1) A+(2-\gamma) \eta]-\eta \gamma^{2} A(A-\eta)=0 .
$$

To show that $(d V / d A)_{+}$vanishes only once in the interval $(\eta, 2 \eta)$, we note that for $\gamma>2$

$$
N(-\infty)>0, \quad N(0)<0, \quad N(\eta)>0, \quad \text { and } \quad N(2 \eta)<0 .
$$


For $1<\gamma<3$ we find that

but

$$
\frac{d}{d A}\left[\frac{(\gamma-1) A+2 \eta}{A-\eta}\right]=-\frac{(\gamma+1) \eta}{(A+\eta)^{2}}<0
$$

$\begin{aligned} \frac{d}{d A}\left\{\frac{\eta \gamma^{2} A}{(2 \eta-A)[(\gamma-1) A+(2-\gamma) \eta]}\right\} & =\frac{\eta \gamma^{2}\left[2(2-\gamma) \eta^{2}+(\gamma-1) A^{2}\right]}{(2 \eta-A)^{2}[(\gamma-1) A+(2-\gamma) \eta]^{2}} \\ >\gamma^{2} \eta^{3}(3-\gamma)(2 \eta-A)^{-2}[(\gamma-1) A+(2-\gamma) \eta]^{-2}>0 & \end{aligned}$

since $\eta<A$ and $\gamma<3$. The function in the braces of (12) is monotone decreasing with A and can vanish at most once in the interval $(\eta, 2 \eta)$; hence $(d V / d A)_{+}$can vanish at most once in $(\eta, 2 \eta)$. Therefore, we have

$$
\begin{array}{rlc}
\left(\frac{d V}{d A}\right)_{+}>0 & \text { for } & \eta<A<A_{e} \\
=0 & A=A_{c}, \\
<0 & A_{c}<A<2 \eta,
\end{array}
$$

where $A_{c}$ is the root of $(12 \mathrm{a})$ in $(\eta, 2 \eta)$. For a backward-facing simple wave where $u+l(A)=$ const and

$$
\left(\frac{d V}{d A}\right)_{-}=\frac{d u}{d A}-\frac{d a}{d A}=-\left(\frac{d l}{d A}+\frac{d a}{d A}\right)=-\left(\frac{d V}{d A}\right)_{+},
$$

just the contrary is true. Nevertheless, since

$$
\left(\frac{d u}{d A}\right)_{+}=\frac{d l}{d A}>0, \quad\left(\frac{d u}{d A}\right)_{-}=-\frac{d l}{d A}<0
$$

it follows from (13) that

$$
\begin{array}{rlc}
\left(\frac{d V}{d u}\right)_{+}>0 & \text { for } & \eta<A<A_{c}, \\
& =0 & A=A_{c}, \\
<0 & A_{c}<A<2 \eta
\end{array}
$$

for both forward-facing and backward-facing simple waves.

TheOREM 4.3. Let $A_{c}$ be the same as that in Theorem 4.2. Then as time increases

(i) in the subregion $\eta<A<A_{c}$ the velocity profile of a simple wave flattens if $u_{x}(x, 0)>0$ but steepens if $u_{x}(x, 0)<0$;

(ii) in the subregion $A_{c}<A<2 \eta$ the velocity profile of a simple wave steepens if $u_{x}(x, 0)>0$ but flattens if $u_{x}(x, 0)<0$.

Proof. Suppose at $t=0$ a simple wave is given with $u=u(x)$ and $a=a(x)$ satisfying the relation $u \mp l(a)=$ const. Since the physical characteristic $C_{\star}$ is straight, the wavelet initiated at a point $x=\zeta$ will propagate at a constant velocity $u(\zeta) \pm a(\zeta)$; its path is given by

$$
x=\zeta+[u(\zeta) \pm a(\zeta)] t
$$

Following the wave, we may consider $u$ as a function of $\zeta$ and in turn as a function of $x$ and $t$ through (14). Thus following the wave and using (14), we obtain

$$
u_{x}=\frac{d u}{d \zeta} \zeta_{x}=\frac{d u}{d \zeta}\left[1+\left(\frac{d u}{d \zeta} \pm \frac{d a}{d \zeta}\right) t\right]^{-1}=\left[\left(\frac{d u}{d \zeta}\right)^{-1}+\left(\frac{d}{d u}\right)_{t} t\right]^{-1}
$$


Note that $u_{x}$ is a measure of the velocity profile and that $d u / d \zeta \equiv u_{x}(x, 0)$. Hence by Theorem 4.2 , the theorem follows immediately.

According to Theorem 4.3, the deformation of a simple wave is affected by its initial wave form and by which of the two subregions it lies. It is conceivable that a steepening simple wave will develop into a shock eventually.

5. Maximum principle. In the region $2 \eta<A$ Eqs. (5) and (6) with $g(A)>0$ and $f(A)<0$ are of elliptic type. Eliminating $u$ from (5) and (6) gives

$$
\begin{aligned}
A_{\imath t}+2 u A_{t x}+\left(u^{2}-f g\right) A_{x x}-\left(1+g^{\prime}\right) g^{-1}\left(A_{t}\right)^{2} \\
-2 u\left(1+g^{\prime}\right) g^{-1} A_{t} A_{x}-\left(g f^{\prime}+f^{\prime}+u^{2} g^{-1}+u^{2} g^{\prime} g^{-1}\right)\left(A_{x}\right)^{2}=0 .
\end{aligned}
$$

For every given solution $A(x, t)$ and $u(x, t)$, the derivatives $A_{x}$ and $A_{t}$ are also given functions of $x$ and $t$; hence the above equation is equivalent to

$$
A_{t t}+C_{1}(x, t) A_{t x}+C_{2}(x, t) A_{x x}+C_{3}(x, t) A_{t}+C_{4}(x, t) A_{x}=0,
$$

and any given solution $A$ may be regarded as a solution of the equivalent linear elliptic equation (15). Similarly, any solution $u$ may be regarded as a solution of an equivalent linear equation like (15). By the maximum principle for linear elliptic equations [8], the maximum of $A$ or $u$ appears on the boundary of its domain of definition.

Lemma. Suppose a solution of (15) $A=A(x, t)$ is of class $C^{2}$ in a closed simply connected region. Then if $A$ assumes a maximum at a boundary point, the inward normal derivation at this point is negative unless $A$ is constant [9].

With these preparations we may state the main theorems for instability as follows.

TheOREM 5.1. Let $u=u(x, t)$ and $A=A(x, t)$ be a non-trivial solution of (5) and (6) in the half plane $t \geqq 0$; let $u_{0}(x)$ denote $u(x, 0)$ and $A_{0}(x), A(x, 0)$. Suppose $u$ and $A$ are of class $C^{2}$ and

$$
u_{0}(x) A_{0}^{\prime}(x)+g\left(A_{0}\right) u_{0}^{\prime}(x) \leqq 0 .
$$

Then either

$$
\lim _{t \rightarrow \infty} A\left(x^{\prime}, t\right) \rightarrow \infty \quad \text { for some } \quad x^{\prime}
$$

or

$$
\lim _{|x| \rightarrow \infty} A\left(x, t^{\prime}\right) \rightarrow \infty \quad \text { for some } t^{\prime}>0 .
$$

Proof. Since $u$ and $A$ are of class $C^{2}$, any given solution $A(x, t)$ may be regarded as a solution of (15). Consider a rectangular region $0 \leqq t \leqq t_{1}$ and $-x_{1} \leqq x \leqq x_{1}$. By the maximum principle and the Lemma, the maximum of $A$ occurs on the boundary except along $t=0$ since $u_{t}(x, 0)=-\left[u_{0}(x) A_{0}^{\prime}(x)+g\left(A_{0}\right) u_{0}^{\prime}(x)\right] \geqq 0$ by the hypothesis. Moreover, the Lemma requires that the inward normal derivative of $A$ must be negative at the point of maximum on the remaining three boundaries. This implies that

$$
\max [A]_{0 \leq t \leq t_{1},|x| \leq\left|x_{2}\right|}<\max [A]_{0 \leq \leq_{3},|x| \leq\left|x_{3}\right|}
$$

provided $t_{1}<t_{2}$ and $\left|x_{1}\right|<\left|x_{2}\right|$. Now, let $t_{1}$ and $x_{1}$ tend to infinity. The theorem follows immediately.

THEOREM 5.2. Under the same conditions as stated in Theorem 5.1 except that (16) is replaced by

$$
u_{0}(x) u_{0}^{\prime}(x)+f\left(A_{0}\right) A_{0}^{\prime}(x) \leqq 0
$$


either

or

$$
\lim _{t \rightarrow \infty} u\left(x^{\prime}, t\right) \rightarrow \infty \quad \text { for some } x^{\prime}
$$

$$
\lim _{|x| \rightarrow \infty} u\left(x, t^{\prime}\right) \rightarrow \infty \quad \text { for some } t^{\prime}>0 .
$$

The proof is completely analogous to that of Theorem 5.1.

Conditions (16) and (17) may be regarded as an instability criterion in the following sense. If one of the conditions is satisfied, the plasma cylinder is unstable; if none of them is true, there may exist a stable solution, for it is possible that the maxima of $u$ and $A$ only occur at $t=0$.

Corollary. Suppose $u$ and $A$ are of class $C^{2}$. Then the plasma cylinder is unstable under disturbances which start with the fluid at rest, i.e., $u_{0} \equiv 0$.

To see this, we merely note that $u_{0} \equiv 0$ is a particular case of (16).

Theorem 5.3. A necessary condition for the plasma to achieve stability is

$$
\eta=\left(B_{0} / C_{0}\right)^{2}>1 / 2 \text {. }
$$

Proof. Since in general the plasma cylinder is unstable in the elliptic region, a necessary condition for stability is to stay away from this region initially. This requires

or

$$
A_{0}=a_{0} / a_{0}=1<2 \eta
$$

$$
\eta=\left(B_{0} / C_{0}\right)^{2}>1 / 2 .
$$

6. Hodograph transformation. Equations (5) and (6) are reducible and hence can be made linear through a hodograph transformation in any region where the Jacobian $\mathcal{J}=u_{t} A_{x}-u_{x} A_{t}$ does not vanish. When $\mathfrak{d} \neq 0$, the following are true: $u_{t}=\mathcal{J} x_{A}$, $u_{x}=-g t_{A}$ and $A_{t}=-g x_{u}, A_{x}=g t_{u}$, which, when substituted into (5) and (6), lead to

$$
\begin{aligned}
& u t_{A}-f(A) t_{u}-x_{A}=0, \\
& g(A) t_{\Delta}-u t_{u}+x_{u}=0 .
\end{aligned}
$$

We may also eliminate $x$ to give, assuming legitimacy of the elimination,

$$
g(A) t_{A A}+\left[1+g^{\prime}(A)\right] t_{A}-f(A) t_{u u}=0,
$$

where $g(A)$ and $f(A)$ are given by (7).

Now, let us examine the region where $\mathcal{J}=0$.

Theorem 6.1. Suppose $A>2 \eta$. Then the Jacobian $\mathcal{g}=u_{t} A_{x}-u_{x} A_{t}$ can not vanish over a region $R$ in the $(x, t)$ plane unless $u \equiv$ const and $A \equiv$ const in $R$.

Proof. If the Jacobian vanishes over $R$ but either $u \not \equiv$ const or $A \not \equiv$ const in $R$, we must have $u_{t} A_{x}=u_{x} A_{t}$ on account of Theorem 3.1. Now, multiplying (5) by $-A_{x}$ and (6) by $u_{x}$, adding the resulting equations, and using $u_{t} A_{x}=u_{x} A_{t}$, we obtain

$$
g(A)\left(u_{x}\right)^{2}+[-f(A)]\left(A_{x}\right)^{2}=0,
$$

which implies $u_{x}=0$ and $A_{x}=0$ since $g(A)>0$ and $f(A)<0$ for $A>2 \eta$. By Theorem 3.1 , only trivial solutions exist; or $u \equiv$ const and $A \equiv$ const. This is a contradiction and hence the theorem is proved.

Theorem 6.2. Suppose $\eta<A<2 \eta$. Then the flow in a region where $\mathfrak{d}=0$ is $a$ simple wave or it must be a constant state. 
Proof. Suppose the flow is not a constant state. Then (20) holds; or

$$
\left(u_{x}\right)^{2}=\left(A_{x}\right)^{2} f(A) / g(A) .
$$

Since the condition $\mathfrak{d}=0$ implies that $u$ and $A$ are functionally related, the above equation integrates to

$$
u= \pm \int[f(A) / g(A)]^{1 / 2} d A+\text { const, }
$$

which is identified as a hodograph characteristic on comparing with (11) and (11a). By definition then, the flow is a simple wave.

Hence, excluding simple waves, which can be handled by the theory of characteristics, we may apply the hodograph transformation in most cases. Equation (19) is, however, difficult to solve especially when the initial conditions are complicated. Some means of finding particular solutions of (19) will now be discussed.

THEOREM 6.3. In the transition zone between the elliptic region and the hyperbolic region $A \approx 2 \eta$, (19) can be locally reduced to the Tricomi equation.

Proof. Let

$$
\alpha(A)=\int_{2 \eta}^{A} \exp \left[-\int\left(1+g^{\prime}\right) g^{-1} d A^{\prime}\right] d A^{\prime}
$$

and consider $u$ and $\alpha$ as the new independent variables. Equation (19) is then transformed into

$$
t_{\alpha \alpha}+k(\alpha) t_{u u}=0
$$

where

$$
k(\alpha)=-f g^{-1} \exp \left[2 \int\left(1+g^{\prime}\right) g^{-1} d A\right] .
$$

In the transition zone, $A \approx 2 \eta$ or $\alpha \approx 0 ; k(\alpha)$ may be approximated by $\alpha k^{\prime}(0)$; and the above equation can be approximated by

$$
k^{\prime}(0) \alpha t_{u u}+t_{\alpha \alpha}=0,
$$

which becomes, after a change of variable from $\alpha$ to $\beta=\alpha\left[k^{\prime}(0)\right]^{1 / 3}$,

$$
\beta t_{u u}+t_{\beta \beta}=0,
$$

which is the Tricomi equation.

Hence, in the transition zone approximate solutions of (19) may be obtained by superposing particular solutions of the Tricomi equation, which is the most studied among equations of mixed type.

In general, however, it is inevitable to solve the hodograph equation (19). We shall now propose a method of finding particular solutions of (19) similar to the idea of sigmaintegration [10]. Let us define a new function $s(u, A)$ such that

$$
s_{u}=F(A) t_{A}, \quad s_{A}=G(A) t_{u}
$$

where

$$
\begin{gathered}
F(A)=\gamma \rho_{0} p_{0}^{-1}[(\gamma-1) A+(2-\gamma) \eta]^{-1}\left[(1-\eta)^{\gamma-1} A^{2(\gamma-1)}(A-\eta)^{\gamma+1}\right]^{1 / \gamma}, \\
G(A)=(2 \eta-A) A^{-2} .
\end{gathered}
$$


It can be checked that if $s_{u A}=s_{A u}$ then eliminating $s$ from (21) leads to (19). Hence, suppose $t_{u u}$ and $t_{A A}$ are continuous; then every solution of (21) is a solution of (19).

Before stating the theorems, we will make a few definitions.

Definition. Let $\phi(z)=s(u, A)+i t(u, A)$ be a single-valued function of $z=$ $u+i A$ in a domain $D$ with $s_{u}, s_{A}, t_{u}$, and $t_{A}$ defined in $D$. Then $\phi(z)$ is said to be $F G$ monogenic at a point $z_{0}$ in $D$ if $s(u, A)$ and $t(u, A)$ satisfy (21) in some $\epsilon$-neighborhood of $z_{0}$. The function $\phi(z)$ is $F G$-monogenic in $D$ if it is $F G$-monogenic at every point in $D$.

Definition. The $F G$-derivative of an $F G$-monogenic function $\phi(z)$ is defined by

$$
\phi^{\prime}(z)=s_{u}+i t_{u}=F t_{A}+i G^{-1} s_{\boldsymbol{A}} .
$$

Definition. The $F G$-integral of an $F G$-monogenic function $\phi(z)$ is defined by

$$
\int_{F G} \phi(z)=\int s d u+G t d A+i \int t d u+F^{-1} s d A .
$$

Note that the $F G$-integral is independent of the path of integration because of (21).

Theorem 6.4. Let $\phi(z)=s(u, A)+i t(u, A)$ be an FG-monogenic function with $s$ and $t$ of class $C^{2}$ in a domain D. Then $\phi^{\prime}(z)$ is $F G$-monogenic in $D$.

Proof. Differentiating (21) with respect to $u$ gives

$$
\begin{gathered}
s_{u u}=F(A) t_{\Delta u}=F(A) t_{u \Lambda}, \\
s_{u A}=s_{\Delta u}=G(A) t_{u u}
\end{gathered}
$$

in $D$. The proof follows immediately from the definitions.

Theorem 6.5. The FG-integral of an FG-monogenic function defined over a domain $D$ is $F G$-monogenic in $D$.

Proof. Let $S=\int s d u+G t d A$ and $T=\int t d u+F^{-1} s d A$. We then find that $S_{u}=s=F(A) T_{A}$ and $S_{A}=G(A) t=G(A) T_{u}$ in $D$. Hence the theorem is proved.

THEOREM 6.6. Let $\phi_{1}(z)$ be FG-monogenic in a domain $D_{1}$ and $\phi_{2}(z)$ be FG-monogenic in a domain $D_{2}$; furthermore let $C_{1} \neq 0$ and $C_{2} \neq 0$ be arbitrary constants. Then $\phi(z)=$ $C_{1} \phi_{1}(z)+C_{2} \phi_{2}(z)$ is $F G$-monogenic in $D_{1} \cap D_{2}$.

The proof follows immediately from (21).

Hence, given an $F G$-monogenic function which corresponds to a solution of (19), by Theorems 6.4, 6.5 and 6.6 we may generate other $F G$-monogenic functions and hence obtain other solutions of (19). In particular, $F G$-monogenic functions 1 and $i$ may be $F G$-integrated to yield infinitely many solutions of (19). It should be noted, however, that many of the solutions so obtained may not have physical meanings.

A simple example serves to illustrate the method. For $s=-1$ and $t=0, F G$-integration once gives $s=-u$ and $t=p_{0} \rho_{0}^{-1}(1-\eta)^{(1-\gamma) / \gamma}\left[(A-\eta)^{-1 / \gamma} A^{(2-\gamma) / \gamma}+\right.$ const $]$. We may choose the constant such that

$$
t / t_{0}=(1-\eta)^{1 / \gamma}(A-\eta)^{-1 / \gamma} A^{(2-\gamma) / \gamma}-1,
$$

where $t_{0}$ is a constant. From (18) we find

$$
x=t_{0} u(1-\eta)^{1 / \gamma}(A-\eta)^{-1 / \gamma} A^{(2-\gamma) / \gamma}-x_{0},
$$

where $x_{0}$ is a constant. Expressing $u$ and $A$ in terms of $x$ and $t$, we obtain

$$
\begin{gathered}
u=\left(x+x_{0}\right)\left(t+t_{0}\right)^{-1}, \\
A^{2-\gamma}(1-\eta)(A-\eta)^{-1}=\left[1+\left(t / t_{0}\right)\right]^{\gamma},
\end{gathered}
$$

which is the same solution as (8b) and (9). 
7. Remarks on stability. For a finite disturbance with moderate area increase $A<2 \eta$, the governing differential equations are hyperbolic. Such a disturbance far away from which the plasma is at constant state is necessarily a simple wave [6]. As a simple wave propagates, its wave form changes but its maximum amplitude remains the same. Hence with respect to a single, axisymmetric, concave or convex disturbance with $A<2 \eta$ and a small area gradient in the axial direction, the plasma cylinder may be regarded as stable in the sense that all its physical quantities remain finite. It is conceivable that through the interaction of several simple waves there may result a finite disturbance which bulges into the elliptic region, in which the plasma is unstable. Thus, the larger the value of $\eta$ and hence the smaller the difference between the confining and internal magnetic field, the more stable the plasma cylinder. This means that for the sake of stability the plasma pressure, which is equal to the difference between the confining and internal magnetic pressures, is to be kept low unless very strong magnetic fields are used.

Acknowledgment. The author wishes to thank Professor T. S. Lundgren for suggesting this research and for his advice, guidance and encouragement during the course of the investigation.

\section{REFERENCES}

1. M. N. Rosenbluth, The stabilized pinch, Proc. Venice Conference on Ionization Phenomena in Gases, pp. 903-907 (1957)

2. S. Chandrasekhar, Plasma physics, University of Chicago Press, Chicago, 1960

3. B. R. Suydam, Stability of a linear pinch, Proc. 2nd U. N. Conference on Peaceful Use of Atomic Energy 31 (1958) 157-159

4. E. A. Frieman and R. M. Kulsrud, Problems in hydromagnetics, in Advances Appl. Mech. 5, Academic Press, 1958, pp. 195-231

5. C. W. Chu, Nonlinear wave motion in an infinite cylindrical plasma confined by a magnetic field, $\mathrm{Ph} . \mathrm{D}$. Thesis, University of Minnesota, 1963

6. R. Courant and K. O. Friedrichs, Supersonic flow and shock waves, Interscience Publishers, Inc., New York, 1948

7. L. Bers and L. Nirenberg, Boundary value problems for nonlinear elliptic equations in two independent variables, Proc. International Cong. of Math. 2, Amsterdam, 1954 pp. 84-85

8. E. Hopf, Elementare Bemerkungen über Lösungen partieller Differentialgleichungen zweiter Ordnung vom elliptischen Typus, Sitzber. Preuss. Akad. Wiss. 19 (1927) 147-152

9. E. Hopf, A remark on linear elliptic equations of second order, Proc. Am. Math. Soc. 3 (1952) 791-793

10. L. Bers, Mathematical aspects of subsonic and transonic gas dynamics, Surveys Appl. Mech. 3, John Wiley \& Sons, New York, 1958, pp. 33-34 